\title{
Structural optimization of timber beams with composite materials
}

\author{
D. Bru, F. B. Varona, S. Ivorra \& F. J. Baeza \\ Department of Civil Engineering, University of Alicante, Spain
}

\begin{abstract}
The present paper outlines the application of a genetic algorithm for the structural constrained optimization problem of the reinforcement of timber beams using composite materials. The genetic algorithm uses an objective function with adaptive penalization as well as an adaptive mutation scheme. The aim is to minimize the material cost of strengthening timber beams and the constraints are the ultimate limit states for flexural and shear behaviour and the serviceability limit state of deflection, according to Spanish Technical Building Code. For this purpose different properties are used, such as section geometry, length, timber class and load conditions. The reinforcement solutions have been encoded in a binary database: type of composite material (CFRP or GFRP), reinforcement mechanical properties and geometric configuration. The search space for the minimum cost consists of 65 billion possible solutions. The genetic algorithm has been used for several specific load and geometry cases for glued laminated timber in order to find whether there is a specific reinforcement configuration more feasible for certain loading situations: short or long beams and lower or higher loading increments. Five cases were analysed. In the first three cases, the length of the beams had constant values of 2, 2.5 and $3 \mathrm{~m}$, whereas the value of loading was variable. In the latter case, the value of the load was fixed and the length of the beam was variable. Analysis of the results shows that GFRP reinforcement is more efficient than CFRP for ultimate limit states.
\end{abstract}

Keywords: fibre reinforced polymers, optimization, computation, wood, glulam, glass fibres, carbon fibres. 


\section{Introduction}

Although the use of wood as a building material is ancient, nowadays it still presents good structural qualities compared to steel and concrete materials. Its stress ratio to specific weight makes it suitable for one way floors and, in particular, wood beams without knots or cracks have high values of compressive and tensile strength. However, elastic modulus and shear strength have very low values compared with steel materials (Triantafillou [1]). Nowadays, building reutilization and the current technical standards lead to an increase in service loads and to a decrease of the maximum deflection limits. In order to improve the structural safety and reliability of structural wood systems in existing buildings, composite-based reinforcement techniques are demanded. In this regard, fibre reinforced polymers (FRP) are an adequate alternative to improve structural capacity (Bru et al. [2]). This study is aimed at reaching and optimum design of the CFRP and GFRP reinforcement of wood beams by means of a genetic algorithm. Genetic algorithms (GAs) have been thoroughly used as an alternative to traditional structural optimization methods. GAs were introduced in the $70 \mathrm{~s}$ by Holland [3]. Concerning the optimum design of the reinforcement for retrofitting of concrete structures, GAs have proven to be a robust technique, as shown in Perera and Varona [4]. Contrary to mathematical programming based optimization methods, GAs are of a probabilistic nature. They are able to handle groups of design points simultaneously and it is also possible to handle continuous and discrete variables. Their application does not need any explicit algebraic formulation of the objective functions and constraint functions and since they do not depend on gradient information, it is not necessary to perform a sensitivity analysis of these functions. Those are their principal advantages over mathematical programming based optimization. GAs are based on the Darwinian principle of evolution, in which the most feasible individuals within a generation are most likely to survive and pass their genetic material on to the next generation. Among their principal advantages, GAs are easily implemented and programmed and, given enough computation time, are always capable of reaching the global optimum. This can be justified because, as noted above, they are able to analyse simultaneously a wide range of possible solutions randomly generated, thus being less sensible to converge to local optima. Other heuristic optimization techniques, such as ant colony optimization and particle swarm optimization have not yet proven as robust as GAs for structural optimization.

\section{Material properties}

The study of the mechanical properties of the materials used in this research was based on the standard ASTM D3039/D3039M for GFRP laminates (Bru et al. [5]). These properties were obtained for bidirectional laminate $\left(0 / 90^{\circ}\right.$ fibre orientation) and for different values of dosage. In this research, weight dosage values of 1/1 were selected, for woven type E glass fibre with $440 \mathrm{~g} / \mathrm{m}^{2}$ weight $(213 / 217)$ and an epoxy resin supplied by SICOMIN Composites, type SR 5550 
and SD 5503. The mechanical properties of other GFRP laminates (Table 1), were determined from the rule of mixtures, eqns (1), (2):

$$
\begin{gathered}
V_{f 1}=\frac{w_{f 1}}{w_{f 2}} \cdot V_{f 2} \\
e_{r}=\frac{N_{d}}{23.75 \cdot\left(\frac{E_{m}}{E_{f}} \cdot \frac{V_{m}}{V_{f}}+1\right) \cdot f_{u, f i b r e}} \cdot\left(\frac{V_{m}}{V_{f}}+1\right)
\end{gathered}
$$

where $V_{f}$ and $V_{m}$ are the volume fraction of the glass fibre woven and the matrix, respectively; $w_{f}$ is the weight of the fabric; $E_{m}$ and $E_{f}$ are the elastic moduli of matrix and fibre materials, respectively; $f_{u, f i b r e}$ is the glass fibre tensile strength; $N_{d}$ is the design axial strength; and $e_{r}$ is the equivalent thickness of the laminate. The units in eqn (2) are mm, $\mathrm{N}$ and $\mathrm{MPa}$.

Table 1: Mechanical properties for CFRP and GFRP laminates.

\begin{tabular}{|c|c|c|c|c|c|c|c|c|}
\hline Material & Fibre weight & Orient. & $e_{r}$ & $V_{f}$ & $V_{m}$ & $E$ & $\sigma_{r t \text { laminate }}$ & $N_{d}$ \\
\hline- & $\mathrm{g} / \mathrm{m}^{2}$ & ${ }^{\circ}$ & $\mathrm{mm}$ & $\%$ & $\%$ & $\mathrm{~N} / \mathrm{mm}^{2}$ & $\mathrm{~N} / \mathrm{mm}^{2}$ & $\mathrm{~N}$ \\
\hline GF-ULE & 630 & 0 & 0.797 & 37.2 & 62.8 & 27823 & 426 & 8070 \\
\hline GF-BXE & 300 & +-45 & 0.718 & 17.7 & 82.3 & 14721 & 225 & 3843 \\
\hline GF-BXE & 315 & +-45 & 0.724 & 18.6 & 81.4 & 15317 & 234 & 4035 \\
\hline GF-BXE & 446 & +-45 & 0.765 & 26.4 & 73.6 & 20518 & 314 & 5713 \\
\hline GF-BXE & 600 & +-45 & 0.793 & 35.5 & 64.5 & 26632 & 407 & 7686 \\
\hline GF-BXE & 800 & +-45 & 0.815 & 47.3 & 52.7 & 34573 & 529 & 10248 \\
\hline GF-BE & 440 & $0 / 90$ & 0.671 & 13.0 & 87.0 & 11545 & 176 & 2818 \\
\hline GF-BE & 600 & $0 / 90$ & 0.718 & 17.7 & 82.3 & 14721 & 225 & 3843 \\
\hline GF-BE & 850 & $0 / 90$ & 0.760 & 25.1 & 74.9 & 19684 & 301 & 5444 \\
\hline CARBODUR & $* *$ & 0 & $*$ & 70.0 & 30.0 & 170000 & 2300.00 & - \\
\hline
\end{tabular}

*Width-thickness values (mm): (50-1.2), (80-1.2), (100-1.4), (120-1.4).

**Weight-thickness values $\left(\mathrm{g} / \mathrm{m}^{2}\right)$ : (1860-1.2), (2170-1.4).

In Table $1, E$ is the elastic modulus of the equivalent laminate and $\sigma_{r t \text {,laminate }}$ is the tensile strength of the equivalent laminate. The values of the elastic moduli of the matrix and fibres are $2810 \mathrm{MPa}$ and $70000 \mathrm{MPa}$, respectively. The mechanical properties of CFRP plates and epoxy matrix have been obtained from SIKA (Carbodur and Sikadur 30). The CFRP plates have standard widths of $50 \mathrm{~mm}$ (model 512E), $80 \mathrm{~mm}$ (model 812E), $100 \mathrm{~mm}$ (model 1014E) and $120 \mathrm{~mm}$ (model 1214E). Finally, the elastic properties of the timber beams were obtained from the Spanish standard, DB SE-M, and plastic properties from previous research [6]. In this way, average value of ultimate compression strain for timber beams is taken equal to 0.01. Currently, the software is only 
implemented with homogenous and nonhomogeneous glulam properties, but different kind of wood and reinforcement materials will be available shortly. Other important property used in the genetic algorithm is the material price and information from Sika, Mel Composites and SICOMIN Composites is used. For Sicomin epoxy resin, $34.5 € / \mathrm{m}^{2}$ has been considered. For glass fibre fabric, the following values have been considered: ULE630, $1.81 € / \mathrm{m}^{2} ;$ BXE300, $1.62 € / \mathrm{m}^{2}$; BXE315, $2.29 € / \mathrm{m}^{2}$; BXE446, $1.98 € / \mathrm{m}^{2}$; BXE600, $2.33 € / \mathrm{m}^{2}$; BXE800, $2.92 € / \mathrm{m}^{2}$; BE440, $1.67 € / \mathrm{m}^{2} ; \mathrm{BE} 850,2.74 € / \mathrm{m}^{2}$. In the case of carbon fibre laminates, the following values were considered: Carbodur 512E, $31.00 € / \mathrm{m}$; Carbodur 812E, $47.10 € / \mathrm{m}$; Carbodur 1014E, $69.10 € / \mathrm{m}$; Carbodur 1214E, $83.20 € / \mathrm{m}$.

\section{Formulation of the genetic algorithm}

The general formulation of an optimization problem is to minimize an objective function subjected to a set of constraint functions, as shown in eqns (3), (4). In this case the objective function $f(x)$ is the cost of the reinforcement:

$$
\begin{gathered}
\text { Minimize } f(x)=C_{C F R P}+C_{G F R P} \\
\text { subject to } g_{i}(x) \leq 0, \quad i=1,2, \ldots, 10
\end{gathered}
$$

where $C_{C F R P}$ is the cost of the CFRP, $C_{G F R P}$ is the cost of the GFRP and $g_{i}(x)$ are the constraints considered for this problem.

Constraint $g_{1}(x)$ corresponds to the bending capacity of the wood beam at the point of maximum moment along the reinforced zone. Constraint $g_{2}(x)$ corresponds to the bending capacity at the point of maximum moment along the unreinforced zone. Constraints $g_{3}(x)$ and $g_{4}(x)$ correspond, respectively, to the tension failure of the CFRP laminate and the GFRP sheet used for flexural reinforcement at the point of maximum moment. And additional constraint $g_{5}(x)$ handles the ultimate stress of the CFRP before delamination occurs at the end of the laminate. Constraint $g_{6}(x)$ corresponds to the shear capacity of the reinforced wood beam at the point of maximum shear force near the supports. Constraint $g_{7}(x)$ corresponds to the shear capacity of the wood beam along the zone without shear reinforcement. All of the previous constraints deal with ultimate limit states (ULS). Constraint $g_{8}(x)$ corresponds instead to the serviceability limit state (SLS) of vertical deformations. Constraint $g_{9}(x)$ corresponds to a geometric limitation concerning the available beam width for installing the CFRP laminates (next to one another). Finally, constraint $g_{10}(x)$ corresponds to another geometric limitation concerning the available beam depth for installing the GFRP sheets. Figure 1 shows the locations where some of the previous design constraints are checked.

The genetic algorithm starts with a randomly generated population of 100 individuals $(j=1,2, \ldots, 100)$. Each individual consists of a binary string (Figure 2), that encodes the reinforcement: type and number of CFRP laminates 
and length of the CFRP reinforced zone $\left(L_{C F R P}\right)$; type and number of layers of GFRP sheets, fibre orientation, length and depth of reinforcement $\left(L_{G F R P, 1}\right.$, $L_{G F R P, 2}$ and $h_{G F R P}($ see Figure 1)).

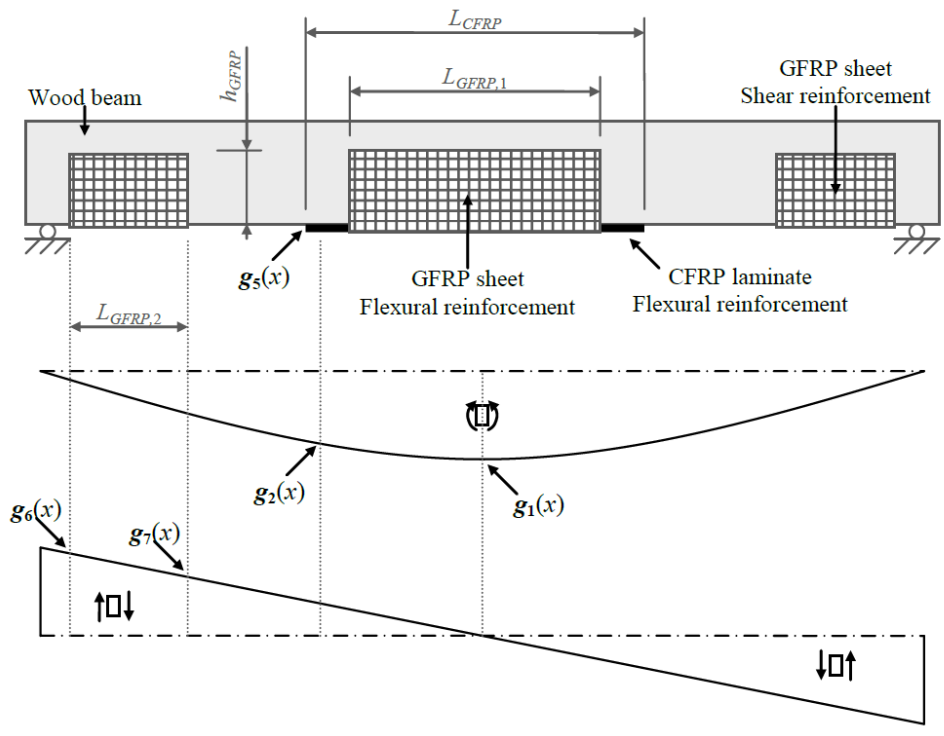

Figure 1: Design constraints associated with flexural and shear ULS.

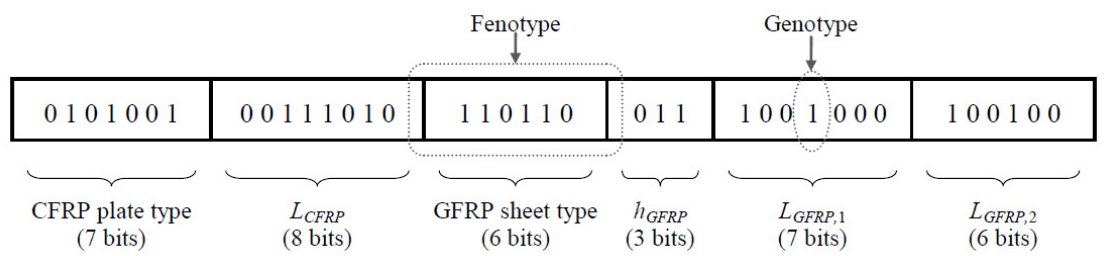

Figure 2: Example of a binary encoded chromosome.

The genetic algorithm needs that the constrained optimization problem is transformed into an unconstrained one. This is done by creating a penalty function $P(x, t)$ which multiplies the objective function. Thus, the new formulation is:

$$
\text { Minimize } \Phi(x, t)=f(x) \cdot P(x, t)
$$

where $t$ is the generation number and $P(x, t)$ is an adaptative penalty function of the type proposed by Gen and Cheng [7] and Perera and Varona [4]. It is calculated through eqn (6): 


$$
\begin{gathered}
P(x, t)=1+\frac{1}{m} \cdot \sum_{i=1}^{m}\left(\frac{\Delta b_{i}}{b_{i}(t)}\right)^{k} \\
\Delta b_{i}=\max \left\{0 ; g_{i}(x)-b_{i}(t)\right\} \quad \text { with } \quad b_{i}(t)=\frac{0,01}{t+1}
\end{gathered}
$$

where a value of $k=2$ has been used in eqn (6). The parameter $b_{i}(t)$ is a penalty threshold which gets lower as the number of generations progresses. When a given individual corresponds to an inexpensive solution which does not satisfy one or several constraints, then its cost gets penalized. Once function $\Phi_{j}(x, t)$ is calculated for each individual, the fitness parameter $S_{j}$ for the $j$-th individual is defined in eqn (8):

$$
S_{j}=1+\frac{\Phi_{\text {min }, t}-\Phi_{j}(x, t)}{\Phi_{\text {max }, t}}
$$

where $\Phi_{\min , t}$ and $\Phi_{\text {max }, t}$ are, respectively the minimum and maximum values of function $\Phi(x, t)$ within the $t$-th generation.

A genetic algorithm has three basic operators: selection, crossover and mutation. The first one, selection, depends on the fitness values; being of a probabilistic nature, a probability of selection for each individual must be defined in eqn (9):

$$
p_{S, j}=\frac{S_{j}}{\sum_{j=1}^{100} S_{j}}
$$

The higher the value of $p_{S, j}$ the more likely will be the $j$-th individual selected for reproduction, thus preserving its genetic material (i.e. reinforcement type and dimensions) on to generation $t+1$. The selected individuals become parents. The crossover (reproduction) operator is performed on a pair of parents and consists on combining the genetic material of the parents, creating two new individuals (possible optimum solutions) which are designated as children.

For this particular genetic algorithm a reproduction probability of $70 \%$ has been used, which means that in some cases (30\% approximately) the genetic material of the parents will not be combined and the pair of children will be identical to the pair of parents. In the rest of cases, the combination of genetic material is made randomly through two different methods: fenotype crossover and flat crossover (Radcliffe [8]).

Mutation of genetic material is not likely to produce more feasible children than their parents, but mutation must be used to avoid convergence to local optima. For the first 10 generations mutation probability varies from $20 \%$ to $15 \%$; between the 11-th generation and the 50-th generation, mutation decreases 
from $15 \%$ to $5 \%$; from the 51 -th generation onwards, mutation is set at a minimum of $5 \%$. However, an adaptative mutation scheme has been programmed when the GA detects that there is little genetic diversity within a given generation (this could happen when converging to a local optimum). The genetic diversity $D(t)$ of generation $t$ is defined in eqn (10):

$$
D(t)=\frac{\Phi_{\text {max }, t}}{\Phi_{\text {mean }, t}}
$$

where $\Phi_{\text {mean, } t}$ is the mean value of the penalized objective function of the 100 individuals of the $t$-th generation. Depending on $D(t)$, mutation probability will corrected as shown in eqn (11):

$$
\text { mutation probability }=\left\{\begin{array}{cll}
13 \%, & \text { if } D(t)=1 \\
10 \%, & \text { if } 1<D(t) \leq 1,05 \\
8 \%, & \text { if } 1,05<D(t) \leq 1,1
\end{array}\right.
$$

Finally, an elitism criterion has been applied, which means that generation $t+1$ will be formed by the best individuals from the group that includes the parents selected from generation $t$ and their children (i.e., if a child is not a better solution for the optimization problem than a given parent, it does not enter generation $t+1$ ).

\section{Design constraints}

To perform this research, two standards have been used to study the bending and shear behaviour of reinforced timber beams, the Spanish standard, DB SE-M and the Italian standard, CNR DT201, and several previous research works, especially for the delamination failure. These design recommendations are based on limit-states design principles and allow compression ductility of the material. This approach sets acceptable levels of safety against the occurrence of both serviceability limit states and ultimate limit states. To assess these limit states, certain values for the design loading and the design strength of the materials must be assumed. Load factors and strength reduction factors stated in Spanish standard. For example, eqn (12), shows the bending strength value of the algorithm for timber beams, $f_{w d}$.

$$
f_{w d}=k_{m o d} \cdot k_{s y s} \cdot k_{h} \cdot \frac{f_{w k}}{\gamma_{m}}
$$

In this case, the program automatically selects the characteristic strength $f_{w k}$ of the wood according to its strength class. The software also selects the correct load factor $k_{\text {mod }}$ according the load duration, the load sharing factor $k_{s y s}$, the height factor $k_{h}$ and the material partial safety factor $\gamma_{m}$. Other factors related to the type of load (dead load, variable load, etc.) are also used in the software. 
According to the previous section, constraints $g_{1}(x)$ and $g_{2}(x)$ correspond to the bending capacity. The following assumptions have been considered. The timber beams are an isotropic material, having tree planes of symmetry. Shear effect is neglected and plane sections remain plane. The stress-strain relationships assumed for wood are elasto-plastic for compression stress and linear-elastic for tension stress. In the case of GFRP and CFRP, linear-elastic behaviour is considered for both compression and tension stress. For FRP reinforced timber beam under the action of a moment, Figure 3 shows the stress and strain distributions of the composite cross-section. The ultimate bending moment of the section $M_{u}$ has been obtained assuming strain compatibility and equilibrium of internal forces and moments; in Figure $3 e_{r G}$ and $e_{r C}$ are the laminate thickness for GFRP and CFRP, respectively; $\sigma_{r c G}$ is the compression stress in the GFRP; $\sigma_{r t G}$ and $\sigma_{r t C}$ are the tensile stresses in the GFRP and in the CFRP laminate, respectively.

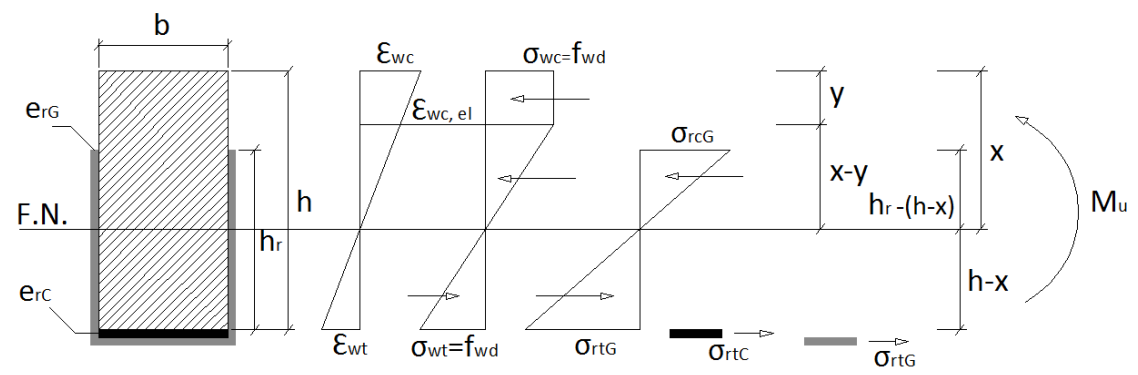

Figure 3: Ultimate moment capacity of FRP strengthened beams.

Constraints $g_{3}(x)$ and $g_{4}(x)$ correspond to the tension failure of laminate. In this case, the ultimate tensile strain of wood is equal to the maximum value of the laminate strain. Therefore, the stress level of the laminates will be around $14 \%$ of its ultimate stress. Thus, these constraints are not likely to be active during the optimization process. However, in future improvements on the algorithm, voids and defects in the wood, could change this hypothesis.

Constraints $g_{5}(x), g_{6}(x)$ and $g_{7}(x)$ correspond to the shear capacity of wood beams. In these cases, two different analyses have been performed. On the one hand, the value of resisting shear force $V_{R d}$ was calculated by transforming the FRP reinforcement to equivalent wood, according to Triantafillou [1], and using the formulation of Collignon-Jourawski and the ultimate strength according to the Spanish standard. In the equivalent section of wood $e_{h r G}$ value is equal to $e_{r G}$ multiplied by the ratio of the laminate elastic modulus to the wood elastic modulus. In this case, it has been assumed that no other failure mechanism such as FRP shear failure occurs prior to wood shear failure. On the other hand, the second possible failure mechanism is due to debonding. In this case, the maximum laminated normal stress $\sigma_{r t C, \max }$ was calculated according to Juvandes and Barbosa [9], as shown in eqn (13): 


$$
\sigma_{r t C, \max }=\frac{c_{1} \cdot k_{b} \cdot k_{c} \cdot \sqrt{E_{r C} \cdot e_{r C} \cdot f_{w t m, p}} \cdot k_{\mu}}{e_{r C}}
$$

where $c_{1}, k_{c}$ and $k_{\mu}$ are experimental factors equal to $0.76,1$, and 1 , respectively; $k_{b}$ is a geometrical function, and $f_{w t m, p}$ is the ultimate pull-off stress, equal to 2.5 MPa. Currently, the software considers the maximum anchor length, according to the properties of the reinforcement. Moreover, this check is performed only for CFRP laminates, due to the lack of information about experimental test for debonding in wrap beams with GFRP.

In addition to the ultimate limit states, the serviceability of a member under service loads should satisfy the provisions of Spanish standard DB SE-M. According to the specifications of the previous standards, constraint $g_{8}(x)$ corresponds to the maximum value of vertical deformations for the following three cases: structural integrity, user comfort and functionality. The calculation method of these three different provisions is indicated in the Spanish standard.

Finally, constraint $g_{9}(x)$ corresponds to the ratio between the total width of the CFRP plates and that of the timber beam cross-section, $b$. In the same way, constraint $g_{10}(x)$ corresponds to the ratio between the perimeter of GFRP and the roll width of fibreglass fabric.

\section{$5 \quad$ FRP design optimization examples}

To evaluate the efficiency of designing FRP for the flexural and shear strengthening of timber beams using GAs and considering the constraints presented previously, four examples are shown. In the first three examples, the length of the beams had constant values of $3,2.5$ and $2 \mathrm{~m}$, whereas the value of loading was variable. In the latter case, the value of the load was fixed to $6.49 \mathrm{kN} / \mathrm{m}$, and the length of the beam was variable. A glulam timber beam GL24h, with a cross section of section $80 \times 160 \mathrm{~mm}$ (width $\times$ depth), was used in all cases. The first value of load was selected equal to the ultimate load of the unreinforced beam. Table 2 shows the optimum reinforcement schemes for the ultimate load, after 10 runs of the genetic algorithm according to the ULS or ULS and SLS together. Figures 4 and 5 show the evolution of the reinforcement unit price according to the load level, for the first two cases. The unit price has been evaluated according to the price of the unreinforced beam. Moreover, the type of reinforcement is showed in blue or grey according to bending or shear failure mode.

Example 1 is a beam with a span length of $3 \mathrm{~m}$ and an initial load of $2.47 \mathrm{kN} / \mathrm{m}$, considering both ULS and SLS design. In this case, the software shows that the optimum solution would consist of one layer of CFRP laminate type $512 \mathrm{E}$ in the middle of the beam covering $42 \%$ of the span length, and three layers of GFRP wrap type ULE630 with 44\% of the total length. However, in the case of considering only ULS, the algorithm would evolve to a solution with 


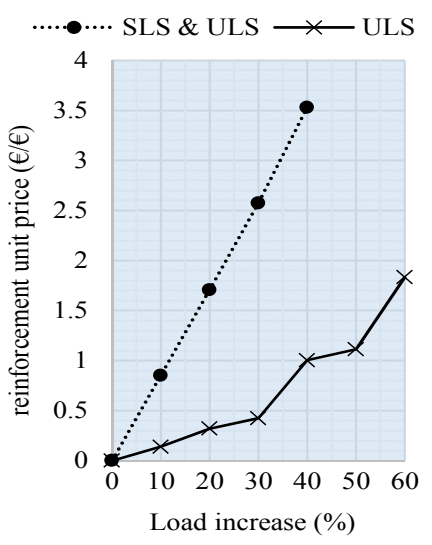

(a)

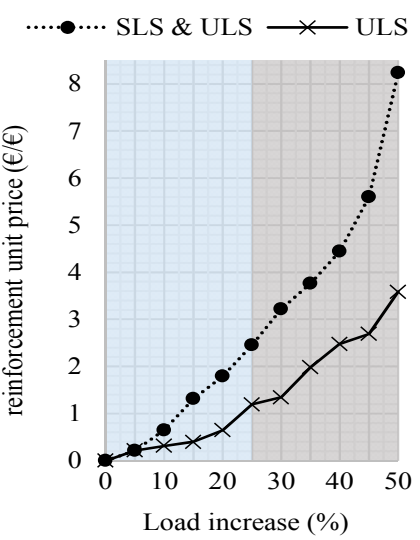

(b)

Figure 4: (a) Example 1. (b) Example 2. In the blue area only flexural reinforcement is needed. In the grey area shear reinforcement is needed.

Table 2: $\quad$ Reinforcement schemes for the final state of loads.

\begin{tabular}{|c|c|c|c|c|c|c|c|c|c|}
\hline & & \multicolumn{3}{|c|}{ CFRP } & \multicolumn{5}{c|}{ GFRP } \\
\hline Example & LS & Layers & $\mathrm{b}_{\mathrm{c}}$ & $\mathrm{L}_{\mathrm{C}}$ & Layers & Type & $\mathrm{H}_{\mathrm{G}}$ & $\mathrm{L}_{\mathrm{G} 1}$ & $\mathrm{~L}_{\mathrm{G} 2}$ \\
\hline- & - & - & $\mathrm{mm}$ & $\mathrm{M}$ & - & - & $\mathrm{mm}$ & $\mathrm{m}$ & $\mathrm{m}$ \\
\hline \multirow{2}{*}{1} & $\mathrm{U}-\mathrm{S}$ & 1 & 50 & 1.25 & 3 & ULE630 & 160 & - & 1.32 \\
\cline { 2 - 11 } & $\mathrm{U}$ & - & - & - & 3 & ULE630 & 160 & - & 1.32 \\
\hline \multirow{2}{*}{2} & $\mathrm{U}-\mathrm{S}$ & 1 & 80 & 1.53 & 3 & ULE630 & 140 & 290 & 1.58 \\
\cline { 2 - 10 } & $\mathrm{U}$ & 1 & 80 & 0.39 & 3 & ULE630 & 90 & 290 & 1.43 \\
\hline \multirow{2}{*}{3} & $\mathrm{U}-\mathrm{S}$ & - & - & - & 3 & ULE630 & 90 & 310 & 0.91 \\
\cline { 2 - 10 } & $\mathrm{U}$ & - & - & - & 3 & ULE630 & 90 & 310 & 0.89 \\
\hline \multirow{2}{*}{4} & $\mathrm{U}-\mathrm{S}$ & 1 & 50 & 1280 & 3 & ULE630 & 90 & 280 & 1.34 \\
\cline { 2 - 10 } & $\mathrm{U}$ & - & - & - & 3 & ULE630 & 90 & 280 & 1.34 \\
\hline
\end{tabular}

GFRP reinforcement only, reducing the cost by $52 \%$, and increasing the ultimate load by $20 \%$. For this reason, in bending problems with SLS limitations, the most important mechanical property is the inertia of the equivalent section, and therefore, the optimal reinforcement schedule will be with CFRP laminates in the middle section. Finally, the horizontal lines of the Figure 4, show that the same scheme of reinforcement can withstand two different levels of load. Notice that no shear reinforcement has been necessary next to the supports.

Example 2 is a beam with a span length of $2.5 \mathrm{~m}$ and an initial load of $4.125 \mathrm{kN} / \mathrm{m}$. In this case, the behaviour of the structure for loads between 0 and $25 \%$ is a flexural reinforcement problem; the solution would be a mixed reinforcement scheme in the mid-span, similar to Example 1, with one layer of CFRP laminate with length equal to $40 \%$ and two layers of GFRP wrap covering to $43 \%$ of the whole beam length. However, for a load increase higher than $25 \%$ 
of the original load, shear reinforcement is necessary next to the supports is needed (see Table 2): in this case, ULE630 shear reinforcement with a length of $290 \mathrm{~mm}$ on each end of the beam ends is necessary for ULS/SLS to withstand an ultimate load increase of $50 \%$ over the original load.

Example 3 is a bending problem with a span length of $2.5 \mathrm{~m}$ and an initial load of $4.125 \mathrm{kN} / \mathrm{m}$. In this case, the behaviour of the structure for loads between 0 and $25 \%$ is a flexural reinforcement problem; the solution would be a mixed reinforcement scheme in the mid-span, similar to Example 1

Example 3 is a beam with a span length of $2 \mathrm{~m}$ and initial load of $6.5 \mathrm{kN} / \mathrm{m}$. In this case, activating or deactivating the SLS design does not alter the optimum reinforcement solution (see Figure 5(a)) and the evolution for ULS only and ULS plus SLS are equal. The reason is that in cases of short beams, the problem of vertical deflection is not a significant constraint and the design is controlled by ULS. In particular, for a load increase of $5 \%$, the algorithm determines the need to reinforce the ends of the beams for shear with one layer of GFRP wrap type BXE300 with $8 \%$ of the total length and $90 \mathrm{~mm}$ of the section. For higher loads, the genetic algorithm evolves to both shear and flexural reinforcements. On the contrary to Examples 1 and 2, the optimum reinforcement uses only GFRP (see Table 2).

Finally, the objective of Example 4 is to check the behaviour of the algorithm in cases of change in length of the beam. In particular, the initial span length is equal to $2 \mathrm{~m}$ and the value of the load is constant to $6.5 \mathrm{kN} / \mathrm{m}$. Figure $5(\mathrm{~b})$, shows two types of behaviour. First, the results for values between 0 to $10 \%$, show the same behaviour as Example 3. In this case SLS constraints are not active. However, for higher lengths, the main problem is the deflection, and the algorithm recommends the use of CFRP laminates for controlling the SLS.

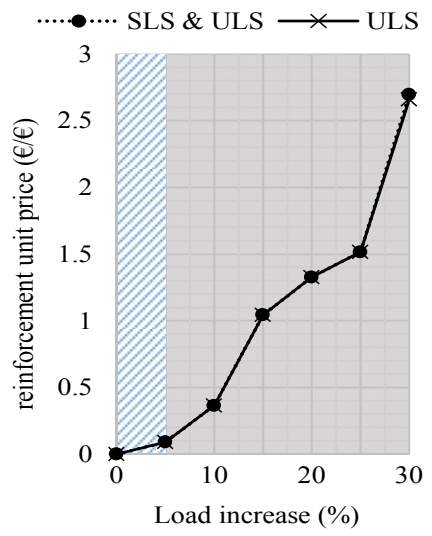

(a)

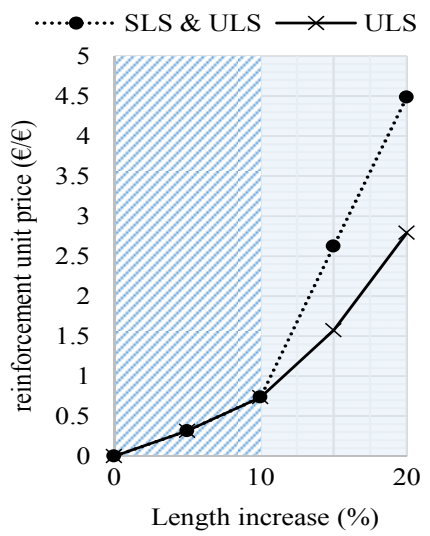

(b)

Figure 5: (a) Example 3. (b) Example 4. Hatched area corresponds to shear reinforced designs. Grey area corresponds to shear and flexural reinforced designs. Blue area corresponds to SLS controlled designs. 


\section{Conclusions}

A genetic algorithm for optimum design of bending and shear FRP reinforcement of low cost timber beams has been presented. This GA serves the purpose of minimizing the material cost associated with the reinforcement materials for ULS and SLS requirements according to the Spanish standards. Finally, the analysis of the 4 examples shows that for structures with flexural failure behaviour, the ultimate load can be increased up to $50-60 \%$ over the ultimate load for the unreinforced beam. However, for beams with shear failure controlled behaviour, the ultimate load should not be increased over $30 \%$ of the unreinforced original load, due to the failure on the supports area.

\section{Acknowledgements}

The authors express deep gratitude to Concepción Puentes Ibáñez and the GRESMES Research Group at the University of Alicante for their useful assistance. This work was partially financed by the University of Alicante by means of the GRE12-04 Research Project and Generalitat Valenciana, grant GV/2014/079.

\section{References}

[1] Triantafillou, T.C. Shear Reinforcement of Wood Using FRP Materials. Journal of Materials in Civil Engineering, 9(2), pp. 22-25, 1997.

[2] Bru, D. Baeza, F.J. Varona, F.B. García-Barba, J. Ivorra, S., Static and dynamic properties of retrofitted timber beams using glass fibre reinforced polymers. Materials and Structures, 2014.

[3] Holland, J.H., Adaptation in natural and artificial systems, MIT Press: Cambridge, Mass., 1975.

[4] Perera, R. \& Varona, F.B., Flexural and shear design of FRP plated RC structures using a genetic algorithm, ASCE Journal of Structural Engineering, 135(11), pp. 1418-1429, 2009.

[5] Bru, D. Baeza, F.J. Varona, F.B. Ivorra, S., Numerical and experimental evaluation of FRP reinforcement on the mechanical behaviour of timber beams. Proc. of the $16^{\text {th }}$ European Conference on Composite Materials. Sevilla, Spain, 2014.

[6] Yang, Y. Liu, J. Xiong, G., Flexural behaviour of wood beams strengthened with HFRP, Construction and Building Materials, 43, pp. 118-124, 2013.

[7] Gen, M. \& Cheng, R., A survey of penalty techniques in genetic algorithms, Proc. of the 1996 Int. Conf. on Evolutionary Computation, ed. IEEE: Nagoya, pp. 804-809, 1996.

[8] Radcliffe, N.J. Equivalence class analysis of genetic algorithms, Complex Systems, 5, pp. 183-205, 1991.

[9] Juvandes, L.F.P. Barbosa, R.M.T., Bond Analysis of Timber Structures Strengthened with FRP Systems, Strain, An International Journal for Experimental Mechanics. 48, pp. 124-135, 2012. 